\title{
In vivo MRI Study of the Visual System in Normal, Developing and Injured Rodent Brains
}

\author{
Kevin C. Chan, Matthew M. Cheung, Kyle K. Xing, Iris Y. Zhou, April M. Chow, \\ Condon Lau, Kwok-fai So and Ed X. Wu ${ }^{*}$
}

\begin{abstract}
This paper demonstrated our recent use of contrast-enhanced MRI, diffusion tensor/kurtosis imaging, proton magnetic resonance spectroscopy, and functional MRI techniques, for in vivo and global assessments of the structure, metabolism and function of the visual system in rodent studies of ocular diseases, optic neuropathies, developmental plasticity and neonatal hypoxic-ischemic brain injury at 7T. Results suggested the significant values of high-field multiparametric MRI for uncovering the processes and mechanisms of developmental and pathophysiological changes systematically along both anterior and posterior visual pathways, and may provide early diagnoses and therapeutic strategies for promoting functional recovery upon partial vision loss.
\end{abstract}

\section{INTRODUCTION}

$\mathrm{T}$ HE integrity of the precise neuronal connections between eye and brain plays a significant role in the performance of the mammalian visual system. To date, the mechanisms of the formation of retinotopic orders between retina and primary visual targets, and the patterns of primary and secondary neurodegeneration upon ocular diseases and injuries remain controversial $[1,2]$. A major obstacle to research into the developmental processes and new treatments for neurodegenerative diseases in the visual system is the lack of a precise and sensitive technique for directly assessing the spatial and temporal evolutions of both anterior and posterior visual pathways under the same experimental conditions. In this paper, we will demonstrate the use of high-field multiparametric magnetic resonance imaging (MRI) at 7T, to provide in vivo and global assessments of the structure, metabolism and function of the visual system from the anterior chamber, vitreous humor and retina in the eye to the optic nerve $(\mathrm{ON})$, optic chiasm $(\mathrm{OC})$, optic tract (OT), superior colliculus (SC), lateral geniculate nucleus (LGN) and primary visual cortex in rodent studies.

Manuscript received April 1, 2010. This work was supported in part by the Hong Kong Research Grant Council (GRF HKU 7793/08M).

K.C.C., M.M.C., K.K.X., I.Y.Z., A.M.C. and C.L. are with the Laboratory of Biomedical Imaging and Signal Processing and the Department of Electrical and Electronic Engineering, The University of Hong Kong, Pokfulam, Hong Kong (e-mails: kevin_ccw@hku.hk, thew@hkusua.hku.hk, xingkai@eee.hku.hk, iriszhou@eee.hku.hk, aprilcmk@hku.hk, conlau@hku.hk).

K.F.S. is with the Department of Anatomy, The University of Hong Kong, Pokfulam, Hong Kong (e-mail: hrmaskf@hkucc.hku.hk)

E.X.W. is with the Laboratory of Biomedical Imaging and Signal Processing and the Departments of Electrical and Electronic Engineering, Anatomy, and Medicine, The University of Hong Kong, Pokfulam, Hong Kong (*corresponding author to provide phone: (852) 2819-9714; fax: (852) 2819-9711; e-mail: ewu@ @ee.hku.hk).
Despite a relatively low visual acuity, rodents are an excellent model for understanding developmental and pathophysiological changes in the visual system given their relatively short lifespan and rapid growth. Their enhanced crossing of optic nerve fibers to the contralateral posterior visual pathway via the optic chiasm (e.g. $>95 \%$ to the rat contralateral SC [3]) also allows the visual components in the opposite hemisphere to serve as an internal control generally upon unilateral manipulation of the visual system.

1. In vivo retinotopic mapping of superior colliculus by molecular imaging techniques using manganese-enhanced MRI (MEMRI)

The retinocollicular projection between the retina and superficial layers of the rodent SC is a well-established model system to study the cellular and molecular mechanisms of retinotopic map formation in the brain [4]. Despite previous studies demonstrating the capability of blood-oxygenation level dependent (BOLD) fMRI for mapping retinotopic organizations in vivo [5-7], BOLDfMRI cannot be readily used to map submillimeter-scale neural activities due to its relatively broad hemodynamic point spread function extending beyond the neuronally active area [8]. In addition, a large field-of-view for high-resolution mapping of the entire visual pathway is difficult to achieve in fMRI due to the tradeoff of spatial resolution for fast imaging sequences to capture physiological responses with optimal signal-to-noise ratios.

To overcome these problems, we recently used the alternative in vivo molecular imaging technique, which relies on the entrance of $\mathrm{Mn}^{2+}$ ions into voltage-gated calcium channel and fast axonal transport $[9,10]$ rather than hemodynamic responses, for global tract-tracing and in vivo MEMRI analyses of retinotopic orders in the optic nerve fibers and the SC. To test whether there is sufficient sensitivity to detect a segregated amount of nerve fibers in the visual pathway, we examined the properties of anterograde axonal transport upon total and partial transections of optic nerve fibers after intravitreal injection of the $\mathrm{Mn}^{2+}$ contrast agent using 3D T1-weighted imaging of the visual pathways at $200 \mu \mathrm{m}$ isotropic resolution (Fig. 1). In the untreated visual pathway ['Normal' in Fig. 1a $(n=4)$ and left eye-right $\mathrm{SC}$ in Fig. $1 \mathrm{~b}(\mathrm{n}=8)]$, clear $\mathrm{Mn}^{2+}$ contrasts were observed in the entire superficial layers of the SC (arrowheads in Fig. 1a) and the LGN (* in Fig. 1a), whereas upon complete transection of the optic nerve fibers at the brachium of SC $(n=8)$, no apparent signal enhancement was observed in the $\mathrm{SC}$, and the $\mathrm{Mn}^{2+}$ ions accumulated proximal 
to the transection site (arrows in Fig. 1a). Upon partial transection of right superior intraorbital optic nerve (arrowhead in Fig. 1b, n=7), the lateral region of the left SC (solid arrow in Fig. 1b) showed a significantly lower signal intensity compared to the medial left SC and the control right SC $(p<0.05)$. A clear border was also observed separating the lateral and medial halves of the left SC, which appeared to colocalize with the topographic projections of the retinocollicular axons relative to the edge of the partial transection in the optic nerve. Note also the signal reduction in the right LGN and optic tract, and the left optic nerve distal to the partial transection. It has been demonstrated that partial transection of the superior intraorbital optic nerve resulted in primary injury predominantly in the superior but not inferior retina and optic nerve [11]. In addition to optic nerve crush [12] and radiation-induced optic nerve injury [13], our previous MEMRI study using a rat model of chronic ocular hypertension also showed a reduced rate of $\mathrm{Mn}^{2+}$ transport in the glaucomatous prechiasmatic optic nerve upon intravitreal $\mathrm{Mn}^{2+}$ injection, in association with a diffuse partial loss of retinal ganglion cell axons [14]. The results of this study demonstrated the sensitivity of submillimeter-resolution MEMRI for in vivo, 3D mapping of the precise retinotopic projections in SC upon reduced anterograde axonal transport of $\mathrm{Mn}^{2+}$ ions from localized regions of the anterior visual pathways. In addition to anterograde, retrograde and transsynaptic degeneration, optic neuropathies are also associated with delayed neuronal death surrounding the lesions in the visual pathways similar to the penumbra in brain injury and ischemia [11]. Further experiments are currently undergoing to determine the course of secondary degeneration surrounding the partial transection at the intraorbital optic nerve, by comparing the signal enhancements in the optic nerve and medial left SC over time. Future experiments may also test the feasibility of activity-induced MEMRI [15] to map retinotopic activities in the visual nuclei.
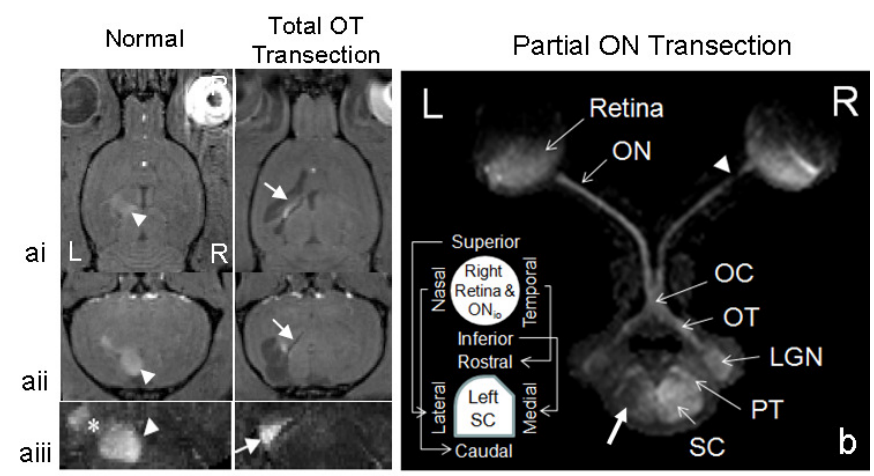

Fig. 1: Typical MEMRI [axial T1WI in (ai); oblique T1WI in (aii); maximum intensity projection of axial T1WI in (aiii) and (b)] of the adult hamster (a) and rat (b) visual pathways without transection [Normal in (a)], with total transection of the left optic tract at the brachium of SC [Total OT transection in (a)], and with partial transection at the right superior intraorbital optic nerve [arrowhead in (b)]. $\mathrm{Mn}^{2+}$ was injected into the right eye only for (a) and into both eyes for (b). Inset in (b) illustrates the graphical representation of retinotopic projection from right retina and intraorbital optic nerve $\left(\mathrm{ON}_{\mathrm{io}}\right)$ to left $\mathrm{SC}$ in rodents. (PT: pretectum)

\section{Microstructural imaging of anterior and posterior visual} pathways using diffusion tensor/kurtosis imaging (DTI/DKI)

In addition to examining neuronal connectivities using MEMRI, in vivo DTI/DKI has been employed to determine the course of microstructural alterations in development and neonatal injury along the visual pathways. Technically, our previous DKI studies using directionally specific kurtosis for probing restricted water diffusion indicated its high sensitivity and comprehensiveness in characterizing brain maturations in the gray and white matters compared to conventional DTI techniques [16, 17]. Mechanistically, using in vivo DTI to a rat model of severe neonatal hypoxicischemic (HI) brain injury covering the entire ipsilesional primary and secondary visual cortices at postnatal day (P) 7 [18], our recent data suggested its feasibility to detect both anterograde and retrograde neurodegeneration along the adult visual pathways at P60.
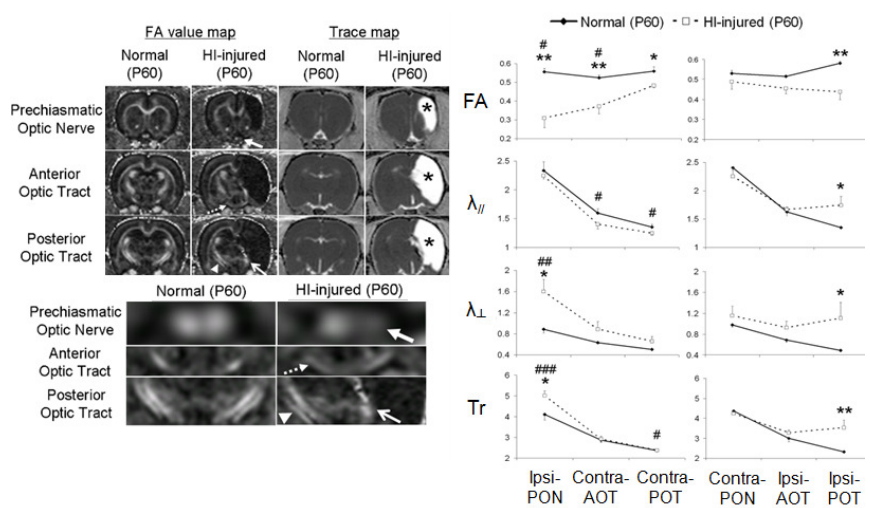

Fig. 2: (Left) FA and diffusion trace maps of a normal rat and an HIinjured rat at P60 at the level of the prechiasmatic optic nerves (PON), and anterior (AOT) and posterior (POT) optic tracts. The expanded views of optic nerves and tracts in the FA map are shown at the bottom. Note the apparent displacement of ipsilesional POT by the porencephalic cyst (asterisks). (Right) Comparisons of DTI parametric values along the visual pathways projected from the ipsilesional (Ipsi-) left eye (left column) and contralesional (Contra-) right eye (right column) in the normal and HIinjured groups. (Units for $\lambda / /, \lambda \perp$ and Tr: $\times 10^{-3} \mathrm{~mm}^{2} / \mathrm{s}$; Two-tailed unpaired t-tests between two groups: $* \mathrm{p}<0.05,{ }^{*} \mathrm{p}<0.01,{ }^{*} * \mathrm{p}<0.001$; Two-tailed paired t-tests between ipsi- and contra-lesional hemispheres in HI-injured group: ${ }^{\#}<<0.05 ;{ }^{\#} \mathrm{p}<0.01 ;{ }^{\# \#} \mathrm{p}<0.001$ in left column)

As shown in Fig. 2, along the visual pathway projected from the ipsilesional eye, the differences in DTI metrics between age-matched controls $(n=7)$ and HI-injured brains at P60 $(n=7)$ were the largest in the ipsilesional prechiasmatic optic nerve (solid arrows) and the smallest in the contralesional posterior optic tract (arrowheads), whereas along the pathway from the contralesional eye, such differences were the largest in the ipsilesional posterior optic tract (open arrows) and the smallest in the contralesional optic nerve. Similar to our previous findings on the DTI changes in other white matter structures [18], it is also believed that the ipsilateral posterior optic tract experienced primary white matter lesion, which was known for reduction in fractional anisotropy (FA), increased average diffusivity (Trace, Tr), and increased axial diffusivity $\left(\lambda_{/ /}\right)$relative to secondary fiber loss resulted from decrease in FA and $\lambda_{/ /}$, and increase in radial diffusivity $\left(\lambda_{\perp}\right)$ and $\operatorname{Tr}$ in the 
ipsilesional optic nerve and contralesional anterior (dashed arrows) and posterior optic tracts [19]. Previous studies showed that visual cortex damage caused trans-synaptic degeneration of the thalamus and the retinal ganglion cells. However, the survivors continued to transmit visual information via the remaining routes to the $\mathrm{SC}$ of the midbrain, which could be enhanced as the result of extensive training [20]. Our results on the long-term outcome of the remaining visual pathways after neonatal brain injury are potentially important in determining and improving the functional consequences of brain lesions after most compensatory and reparative phases have been passed.

3. Evaluation of steady-state metabolisms by ${ }^{1} H$-MRS, and dynamics of blood-tissue metabolite exchanges by gadolinium contrast-enhanced MRI in the visual nuclei

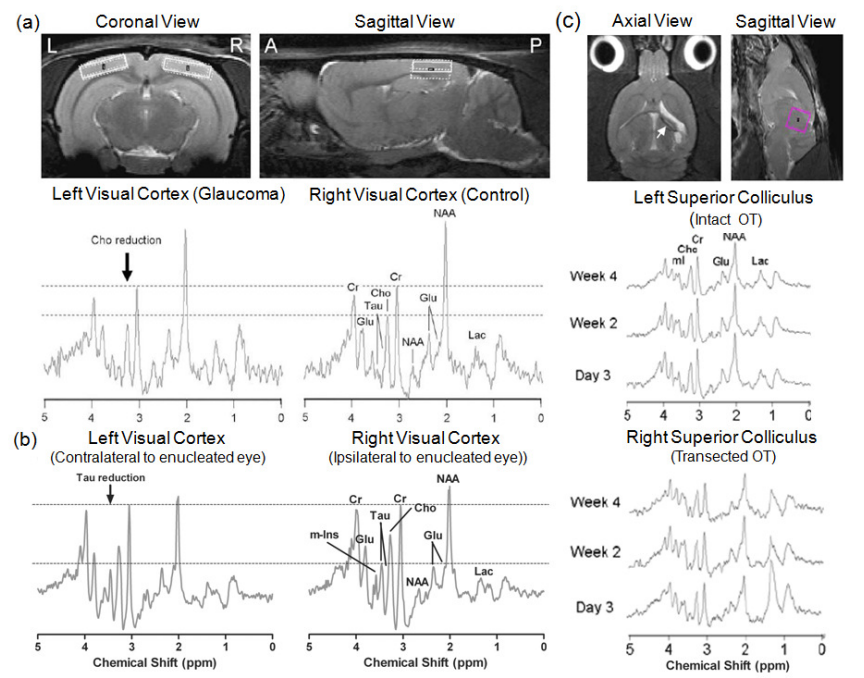

Fig. 3: Single-voxel ${ }^{1} \mathrm{H}-\mathrm{MRS}$ of the visual cortex (a and b) and SC (c) in the posterior visual pathways upon ocular hypertension (a) and neonatal monocular enucleation (b) in the right eye in rats, and upon complete transection of the optic tract fibers at the brachium of SC in hamsters (arrow) (c). Brain images on top of (a) and (c) indicate the localization of voxels (rectangular boxes) for ${ }^{1} \mathrm{H}-\mathrm{MRS}$ acquisitions.

Using localized single-voxel ${ }^{1} \mathrm{H}-\mathrm{MRS}$ covering the rat visual cortex, our previous study indicated its feasibility in detecting alterations in major metabolites (choline and glutamate) upon chronic ocular hypertension in the steady state [21] (Fig. 3a). Recently we extended our method to test its sensitivity in detecting metabolic changes in the primary visual nuclei in other ocular diseases and injuries in relation to vision loss. In a neonatal rat model of monocular enucleation at P10 $(n=8)$, we observed a reduction in taurine level in the visual cortex contralateral to the enucleated eye at juvenile age at P30 (Fig. 3b) in comparison to the agematched control $(n=6)$. In a longitudinal ${ }^{1} \mathrm{H}-\mathrm{MRS}$ study upon deafferentation of the adult hamster optic tract $(n=6)$, a consistent increase in lactate, and a transient decrease in NAA and glutamate followed by a delayed increase in myoinositol were also found in the ipsilesional SC from 3 days to 4 weeks after lesion (Fig. 3c) compared to the contralateral side. Our preliminary study, conducted using the established chronic ocular hypertension model, has also demonstrated for the first time that systemic administration of gadolinium contrast agent could provide early and sensitive detection of enhanced contrast leakage from the ciliary body into the anterior chamber and vitreous humor (Fig. 4) of the glaucomatous eye [22], suggestive of the compromise of blood-aqueous or aqueous-vitreous barrier integrity upon chronic ocular hypertension.

In ocular diseases such as glaucoma, increasing evidence suggested the occurrence of early lesions in both the retina and optic nerve of the anterior visual pathway, as well as the primary visual targets along the posterior visual pathway [2, 23]. Yet the primary sources of injury and their subsequent courses of anterograde, retrograde and transsynaptic neurodegeneration along and surrounding the injury sites in are still unclear. Our current procedures of ${ }^{1} \mathrm{H}-\mathrm{MRS}$ and gadolinium-enhanced MRI at high magnetic field strength may provide a sensitive and non-invasive measure of the metabolic processes and mechanisms of ocular diseases and injuries over time. They may also be potentially applicable to examine at high spectral resolutions the subtle alterations in small metabolites in other visual components, including the vitreous and LGN, and to assess the integrity of bloodaqueous, blood-retinal, aqueous-vitreous, blood-nerve and blood-brain barriers along normal and lesioned visual pathways in neonatal, juvenile and adult subjects.

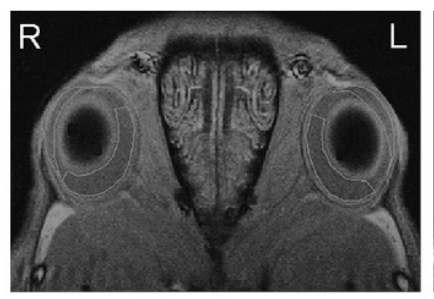

Before injection

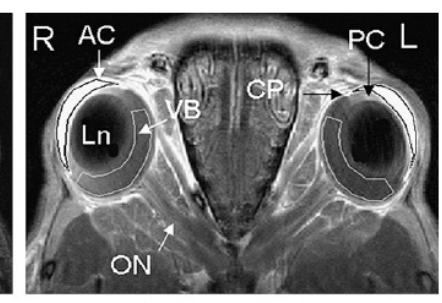

60 mins after injection
Fig. 4: Gadolinium contrast-enhanced-MRI allows visualization of the visual components in the anterior visual pathway, and quantitation of the blood-tissue metabolic exchange dynamics in anterior chamber (AC) and vitreous body (VB) in the left normal and right glaucomatous eyes. [lens $(\mathrm{Ln})$, ciliary processes $(\mathrm{CP})$, posterior chamber $(\mathrm{PC})]$

4. Detection of functional responses in postnatal visual development, developmental plasticity, and brain dynamics in the visual pathways upon visual flash stimulation

In recent years, studies have successfully employed BOLD-fMRI to identify the neurovascular coupling in the retina [24] and the primary visual nuclei [25, 26] in adult rodents. However, the temporal dynamics and interactions among these components during development and plasticity are still largely unknown. In normal adult rats, we have recently observed the temporal differences in BOLD responses among $\mathrm{SC}$, LGN $(\mathrm{n}=15)$, and substantia nigra $(n=12)$, with the SC responding the fastest upon visual flash illumination to either eye (data not shown). In normal visual development, our previous findings upon diffuse visual flash stimulation suggested the presence of neurovascular couplings in the superficial layers of the rat normal SC since eye opening at about P14, as well as its progressive changes in the amplitude and temporal BOLD responses up till adulthood at P60 [27]. Recently we have extended the study 
to monitor subcortical visual responses in severe neonatal HI-injury at P7 involving the left cortex and the SC. Interestingly, while visual stimulation to the right eye gave diminished BOLD responses in the contralateral left SC at P60 as expected [27] for mild lesions to left $\mathrm{SC}(\mathrm{n}=5)$ (Fig. $5 b$ ), in the HI-injured brain involving severe lesions to left SC (Fig. 5c) (n=3), BOLD activations were found in the right $\mathrm{SC}$ upon right eye stimulation. These results indicated the feasibility of BOLD-fMRI to detect developmental plasticity and functional reorganization in the subcortical visual nuclei. (a) Normal brain

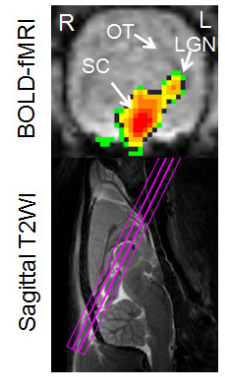

(b) $\mathrm{HI}$-injured brain with mild lesion at left SC
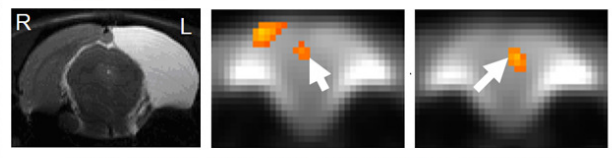

(c) HI-injured brain with severe lesion at left SC

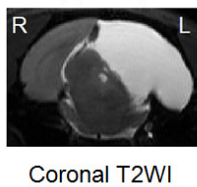

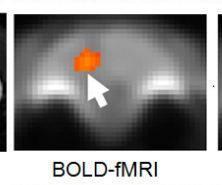

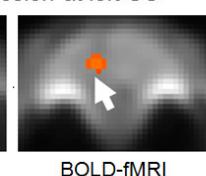

(Left eye stimulation) (Right eye stimulation)

Fig. 5: (a) BOLD-fMRI activation correlation map overlaid onto the singleshot gradient-echo echo-planar images (top), in an oblique orientation parallel to the laminar layers of the rat SC (bottom). Visual flash stimulation was applied to the right eye and activations were predominantly observed in the left LGN and SC. Activations in the visual cortex were also observed occasionally in both hemispheres. Correlation threshold was set at 0.2 . (b,c) Coronal $\mathrm{T} 2$-weighted anatomical images (left column) and activation correlation maps overlaid onto the spin-echo echo-planar images upon left (middle column) and right eye stimulations (right column) to neonatally $\mathrm{HI}$-injured rat brains with left SC barely (b) or severely (c) damaged. Solid arrows indicate the activations at SC. Stimulation to the left/right eye resulted in BOLD activations in the contralateral right/left SC in mild SC lesion in (b). However, the right SC was activated upon right eye stimulation in severe SC lesion in (c).

\section{CONCLUSIONS}

In summary, the preliminary data here have shown the sensitivity of in vivo multiparametric MRI approaches for understanding the structure, metabolism and function of the rodent visual system globally over time at high magnetic field strength. We anticipate that both spatial precision and signal-to-noise ratio can be further improved to determine subregional changes in the visual pathways. Results from these studies should not only improve scientific knowledge in the fields of neuro-ophthalmology, developmental biology, systems neuroscience, and pathophysiology, but our MEMRI study could also push the detection limits for in vivo molecular imaging toward individual nerve fiber characterization at submillimeter ranges in the visual system. Data from the animal studies should open up new areas of research into the organizations of the visual pathway, and lay the foundation for future human studies.

\section{REFERENCES}

[1] A. D. Huberman, M. B. Feller, and B. Chapman, "Mechanisms underlying development of visual maps and receptive fields," Annu Rev Neurosci, vol. 31, pp. 479-509, 2008

[2] N. Gupta and Y. H. Yucel, "Should we treat the brain in glaucoma?," Can J Ophthalmol, vol. 42, pp. 409-13, Jun 2007.

[3] J. Forrester and A. Peters, "Nerve fibres in optic nerve of rat," Nature, vol. 214, pp. 245-7, Apr 151967.
[4] D. D. O'Leary and T. McLaughlin, "Mechanisms of retinotopic map development: Ephs, ephrins, and spontaneous correlated retinal activity," Prog Brain Res, vol. 147 , pp. 43-65, 2005

[5] W. Chen, X. H. Zhu, K. R. Thulborn, and K. Ugurbil, "Retinotopic mapping of lateral geniculate nucleus in humans using functional magnetic resonance imaging," Proc Natl Acad Sci U S A, vol. 96, pp. 2430-4, Mar 21999.

[6] R. O. Duncan, P. A. Sample, R. N. Weinreb, C. Bowd, and L. M. Zangwill, "Retinotopic organization of primary visual cortex in glaucoma: Comparing fMRI measurements of cortical function with visual field loss," Prog Retin Eye Res, vol. 26, pp. 38-56, Jan 2007.

[7] K. A. Schneider, M. C. Richter, and S. Kastner, "Retinotopic organization an functional subdivisions of the human lateral geniculate nucleus: a high-resolution functional magnetic resonance imaging study," J Neurosci, vol. 24, pp. 8975-85, Oct 132004.

[8] S. A. Engel, G. H. Glover, and B. A. Wandell, "Retinotopic organization in human visual cortex and the spatial precision of functional MRI," Cereb Cortex, vol. 7, pp. 181-92, Mar 1997.

[9] R. G. Pautler, "In vivo, trans-synaptic tract-tracing utilizing manganese-enhanced magnetic resonance imaging (MEMRI)," NMR Biomed, vol. 17, pp. 595-601, Dec 2004.

[10] J. E. Merritt, R. Jacob, and T. J. Hallam, "Use of manganese to discriminate between calcium influx and mobilization from internal stores in stimulated human neutrophils," J Biol Chem, vol. 264, pp. 1522-7, Jan 251989.

[11] H. Levkovitch-Verbin, H. A. Quigley, K. R. Martin, D. J. Zack, M. E. Pease, and D. F. Valenta, "A model to study differences between primary and secondary degeneration of retinal ganglion cells in rats by partial optic nerve transection," Invest Ophthalmol Vis Sci, vol. 44, pp. 3388-93, Aug 2003.

[12] M. Thuen, T. E. Singstad, T. B. Pedersen, O. Haraldseth, M. Berry, A. Sandvig, and C. Brekken, "Manganese-enhanced MRI of the optic visual pathway and optic nerve injury in adult rats," J Magn Reson Imaging, vol. 22, pp. 492-500, Oct 2005.

[13] S. Ryu, S. L. Brown, A. Kolozsvary, J. R. Ewing, and J. H. Kim, "Noninvasive detection of radiation-induced optic neuropathy by manganese-enhanced MRI," Radiat Res, vol. 157, pp. 500-5, May 2002.

[14] K. C. Chan, Q. L. Fu, E. S. Hui, K. F. So, and E. X. Wu, "Evaluation of the retina and optic nerve in a rat model of chronic glaucoma using in vivo manganeseenhanced magnetic resonance imaging," Neuroimage, vol. 40, pp. 1166-74, Apr 152008.

[15] I. Aoki, C. Tanaka, T. Takegami, T. Ebisu, M. Umeda, M. Fukunaga, K. Fukuda, A. C. Silva, A. P. Koretsky, and S. Naruse, "Dynamic activity-induced manganese-dependent contrast magnetic resonance imaging (DAIM MRI)," Magn Reson Med, vol. 48, pp. 927-33, Dec 2002.

[16] M. M. Cheung, E. S. Hui, K. C. Chan, J. A. Helpern, L. Qi, and E. X. Wu, "Does diffusion kurtosis imaging lead to better neural tissue characterization? A rodent brain maturation study," Neuroimage, vol. 45, pp. 386-92, Apr 12009.

[17] E. S. Hui, M. M. Cheung, K. C. Chan, and E. X. Wu, "B-value dependence of DT quantitation and sensitivity in detecting neural tissue changes," Neuroimage, vol. 49, pp. 2366-74, Feb 12010.

[18] K. C. Chan, P. L. Khong, H. F. Lau, P. T. Cheung, and E. X. Wu, "Late measures of microstructural alterations in severe neonatal hypoxic-ischemic encephalopathy by MR diffusion tensor imaging," Int J Dev Neurosci, vol. 27, pp. 607-15, Oct 2009.

[19] C. Pierpaoli, A. Barnett, S. Pajevic, R. Chen, L. R. Penix, A. Virta, and P. Basser, "Water diffusion changes in Wallerian degeneration and their dependence on white matter architecture," Neuroimage, vol. 13, pp. 1174-85, Jun 2001.

[20] R. Werth, "Cerebral blindness and plasticity of the visual system in children. A review of visual capacities in patients with occipital lesions, hemispherectomy or hydranencephaly," Restor Neurol Neurosci, vol. 26, pp. 377-89, 2008.

[21] K. C. Chan, K. F. So, and E. X. Wu, "Proton magnetic resonance spectroscopy revealed choline reduction in the visual cortex in an experimental model of chronic glaucoma," Exp Eye Res, vol. 88, pp. 65-70, Jan 2009.

[22] K. C. Chan, Q. L. Fu, H. Guo, K. F. So, and E. X. Wu, "GD-DTPA enhanced MRI of ocular transport in a rat model of chronic glaucoma," Exp Eye Res, vol. 87, pp. 334-41, Oct 2008.

[23] A. J. Weber, C. D. Harman, and S. Viswanathan, "Effects of optic nerve injury, glaucoma, and neuroprotection on the survival, structure, and function of ganglion cells in the mammalian retina," J Physiol, vol. 586, pp. 4393-400, Sep 152008.

[24] T. Q. Duong, M. T. Pardue, P. M. Thule, D. E. Olson, H. Cheng, G. Nair, Y. Li, M. Kim, X. Zhang, and Q. Shen, "Layer-specific anatomical, physiological and functional MRI of the retina," NMR Biomed, vol. 21, pp. 978-96, Nov 2008

[25] C. P. Pawela, A. G. Hudetz, B. D. Ward, M. L. Schulte, R. Li, D. S. Kao, M. C. Mauck, Y. R. Cho, J. Neitz, and J. S. Hyde, "Modeling of region-specific fMRI BOLD neurovascular response functions in rat brain reveals residual differences that correlate with the differences in regional evoked potentials," Neuroimage, vol. 41, pp. 525-34, Jun 2008 .

[26] N. Van Camp, M. Verhoye, C. I. De Zeeuw, and A. Van der Linden, "Light stimulus frequency dependence of activity in the rat visual system as studied with high-resolution BOLD fMRI," J Neurophysiol, vol. 95, pp. 3164-70, May 2006.

[27] K. C. Chan, K. K. Xing, M. M. Cheung, I. Y. Zhou, and E. X. Wu, "Functional MRI of postnatal visual development in normal and hypoxic-ischemic-injured superior colliculi," Neuroimage, vol. 49, pp. 2013-20, Feb 12010. 